\title{
Tuberculous pericarditis, a topical pathology in pediatrics - a case report and a review of the literature
}

\author{
Lorena Elena Melit', Cristina Oana Marginean', George Rolea², \\ Vladut Stefan Sasaran ${ }^{3}$, Cristian Dan Marginean ${ }^{3}$ \\ ${ }^{1}$ Pediatrics Clinic 1, Targu-Mures, Romania \\ 2Obstetrics and Gynecology Clinic 1, Targu-Mures, Romania \\ ${ }^{3}$ University of Medicine and Pharmacy, Targu-Mures, Romania
}

\begin{abstract}
Tuberculosis is one of the most frequent infectious pathologies, representing a major health public problem worldwide, with increased incidence in pediatric ages. We present the case of a 15-year-old male patient, who was admitted in our clinic for fever, malaise, vomiting, productive cough, dizziness and thoracic pain. His personal history underlined the fact that the patient also presented thoracic pain approximately 6 month before, which was interpreted as an intercostal neuralgia. The laboratory tests showed: leukocytosis, neutrophilia, monocytosis, increased inflammatory biomarkers and hyponatremia. The echocardiography revealed pericardial effusion. Despite the wide-spectrum antibiotics, the quantity of pericardial fluid increased, therefore a pericardial puncture was required. Due to the slow favorable evolution and the negative results of the common tests from the puncture fluid, we performed also PCR assays, which identified the bacterial DNA in the fluid establishing the diagnosis of tuberculous pericarditis. The particularity of the case consists in diagnosing a tuberculous pericarditis in a 15-year-old male teenager, with a recent history of thoracic pain, only based on the detection of M. tuberculoris DNA in the pericardial fluid, in the lack of other positive tests for this infection.
\end{abstract}

Keywords: pericarditis, pediatrics, tuberculosis
Abbreviations
Hb: hemoglobin
Htc: hematocrit
CRP: C-reactive protein
ESR: erythrocyte sedimentation rate
H: height
M. tuberculosis: Mycobacterium tuberculosis

\author{
MEV: medium erythrocyte volume \\ PCR: polymerase chain reaction \\ TB: tuberculosis \\ W: weight \\ WHO: World Health Organization
}

\section{INTRODUCTION}

Tuberculosis (TB) is one of the most frequent infectious pathologies worldwide, known for more than 4,000 years. The term of TB was introduced for the first time in 1830 by Schönlein, and comes from the English term "tubercle", which means consumption lesion. The Mycobacterium tuberculosis (M. tuberculosis) complex comprises 5 types of bacteria, namely: M. tuberculosis, Mycobacteri- um africanum, Mycobacterium bovis, Mycobacterium microti and Mycobacterium carnetti, but $M$. tuberculosis is the one that causes most frequently TB in human beings. Mycobacteria grow slowly on culture media, such as Löwenstein-Jensen, at temperatures between 37 and $41^{\circ} \mathrm{C}$ ), isolation on media lasting between 3 and 6 weeks, and susceptibility testing for specific drugs requires another 2-4 weeks. The transmission is by air. World Health Organization (OMS) sustains that TB is the second 
infectious death leading cause worldwide (after HIV), and that approximately one-third of the people from the globe are infected with M. tuberculosis, $95 \%$ of the cases belonging to developing countries (1). Also, OMS states that in 2013, approximately 550,000 new cases of TB were identified in children leading to 80,000 deaths worldwide in non-HIV children (1).

Tuberculous pericarditis is rare, accounting for approximately $0.5-4 \%$ of the TB cases in children, but is the most frequent form of cardiac $\mathrm{TB}$, as a result of direct invasion of lymphatics from the subcarinal lymph nodes (1). The symptomatology is unspecific, and it can include: fever, malaise, weight loss and thoracic pain, less frequent in children (1). The pericardial fluid is sero-fibrinous or hemorrhagic, and the diagnosis is very difficult to be established. The microscopy for detecting Koch bacillus in the puncture fluid is most often negative, and the culture is positive only in $30-70 \%$ of the case (1). Nevertheless, the culture from the pericardial biopsy is a more sensitive diagnosis alternative, and the detection of granulomas suggests the positive diagnosis (1). The polymerase chain reaction type assays detect the bacterial DNA in different biological samples, being a high costing method that can be performed also from peripheral blood, when biological samples from infection situs are not available, but with a decreased sensitivity (2). Tuberculin reaction (IDR) remains one of the most simple and useful diagnostic tools. The TB treatment is very complex and long-lasting, requiring close monitoring from both care-givers and physician. Thus, the prognosis of TB depends mostly on compliance, but also on the potential complications.

\section{CASE REPORT}

We present the case of a 15 -year-old male patient, who was admitted in our clinic for fever, productive cough, rhinorrhea, anterior thoracic pain, dizziness and vomiting. The family history was not relevant for the present pathology. The personal history revealed the fact that patient presented also thoracic pain 6 month before the admission for which he went to the emergency room and was diagnosed with intercostal neuralgia. The present pathology had the onset approximately one week be- fore the admission with fever $\left(38^{\circ} \mathrm{C}\right)$, cough, initially dry, and afterwards productive, nasal obstruction, associating for 2 days intense thoracic pain, accentuated by inspire, dizziness and vomiting, therefore being admitted in our clinic.

The clinical exam from the moment of admission revealed the following pathological elements: malaise, orthopnea, well-represented adipose tissue, saburral tongue, hyperemic pharynx, hypertrophic tonsils, productive cough, nasal obstruction, bleared cardiac sounds, arterial hypotension TA: $86 / 49 \mathrm{mmHg}, \mathrm{O}_{2}$ saturation 92\%, W: $85 \mathrm{~kg}, \mathrm{H}$ : $167 \mathrm{~cm}$.

The CBC count performed at the moment of admission showed: leukocytosis $(13,670 / \mu \mathrm{L})$, neutrophilia $(9880 / \mu \mathrm{L})$, monocytosis $(2,480 / \mu \mathrm{L})$, and anemia (Hb 11.4 g/dL, Htc 33.9\%, MEV 77.8 fL). The biochemistry pointed out hyponatremia (126.9 $\mathrm{mmol} / \mathrm{L})$, and the inflammatory biomarkers were elevated (CRP $136 \mathrm{mg} / \mathrm{l}$, ESR $105 \mathrm{~mm} / \mathrm{h}$ ). We also performed a thoracic radiography which revealed a suggestive aspect for interstitial pneumonia, and echocardiography that showed a pericardial effusion surrounding the heart of approximately $1.7 \mathrm{~cm}$ anteriorly of the right ventricle, and $2.3 \mathrm{~cm}$ posteriorly of the left ventricle, without cardiac tamponade signs, recommending diuretics (Furosemide and Spironolactone) associated with intravenous perfusion for correcting the hyponatremia. The blood culture was negative. We also performed specific serological test excluding an autoimmune pathology. Thus, we administered antibiotics, third generation cephalosporin (Cefort) associated with aminoglycoside (Amikacin) for 5 days, but the fever persisted, therefore we changed the antibiotics with Meropenem and Vancomycin, but we also administered antifungal symptomatic drugs. Despite the diuretic treatment, the echocardiography performed on the $7^{\text {th }}$ day of admission pointed out an increase of the pericardial fluid, therefore pericardial puncture was performed draining approximately $150 \mathrm{ml}$ sero-hemorrhagic fluid. The microbiological exam from the pericardial fluid was negative and likewise the microscopic one for the detection of Koch bacillus, and the pathological exam pointed out only the presence of inflammatory cells. On the $10^{\text {th }}$ day of admission, the patient developed fever again, therefore the carbapenem was replaced by a fluoroquinolone (Levofloxacin), 
a cardiac MRI being also performed, which pointed out only the presence of a retrocardiac thrombus. After initiating the previously mentioned treatment, the evolution was favorable, therefore we performed PCR assays from the pericardial fluid, which detected the presence of M. tuberculosis DNA, and therefore the patient was transferred in the Pneumophtisiology Clinic in order to receive a specific treatment and an adequate monitoring.

The particularity of the case consists in diagnosing a tuberculous pericarditis in a 15-year-old male teenager, with a recent history of thoracic pain, only based on the detection of $\mathrm{M}$. tuberculosis DNA in the pericardial fluid, in the lack of other positive tests for this infection.

\section{DISCUSSIONS}

Tuberculosis is a major health public problem worldwide. In pediatric ages, mortality due to TB is the highest among children under the age of 5 years without an early treatment due to the difficulties related to establishing the diagnosis, but also in those with HIV coinfection despite the adequate treatment (3). Besides the fact that TB diagnosis is difficult to be established in case of children under the age of 5 years, these represent and high risk category also due to the increased susceptibility of developing TB meningitis $(4,5)$. A recent study pointed out that approximately $19.3 \%$ of the children with TB meningitis will die, and $53.9 \%$ of the survivors will present long-term neurological sequelae (6). In Romania, the overall TB incidence decreased progressively, from $47.2 \%_{000}$ in 2002 to $82.8 \%_{000}$ in 2011 , and among children, in the same period, from $47.2 \%_{000}$ (1784 cases) to $23.6 \%_{000}$ (766 cases) (7).

TB pericarditis is a rare pathology in children that involved a variety of difficulties related to establishing the positive diagnosis. The symptomatology of TB pericarditis in children in non-specific including: fever, malaise, weight loss, or thoracic pain, rare in children (1). The patient described above presented fever, malaise, but not weight loss, contrariwise being an obese teenager. Even though in the literature, thoracic pain is reported to be rare in children with TB pericarditis, our patient also presented, in addition to the previously mentioned symptoms, intense anterior thoracic pain. The laboratory tests and the imagistic investigations are often non-specific in case of children affected by B. Two relatively commonly used tests in diagnosing TB are tuberculin reaction and interferon-gamma release assays. In a study performed on 109 Moroccan children the interferon-gamma release assays presented higher sensitivity and specificity in comparison to tuberculin test, therefore being recommended their corroboration in order to establish a more accurate diagnosis (8). The PCR assays identify both the bacterial DNA from different biological samples and the presence of bacterial resistivity features. According to a recent study, these tests detect M. tuberculosis also in the culture-negative cases leading to the conclusion that they are probably the most sensitive and specific tests useful to increase the detection rate (9). Similarly, in the case presented above, the presence of $M$. tuberculosis was also pointed out only by the PCR assays. In addition, besides the very high incidence of this pathology worldwide, the increase of multi-drug resistant cases of TB (MDR) is another concern, being defined as TB caused by strains of $M$. tuberculosis resistant to isoniazid and rifampicin (3). A recent review estimated that approximately 32,000 children are affected by MDR TB (3). Latent TB is another medical entity that deserves increased attention from the pediatrician (10), due to the fact that its annual prevalence in children is of approximately 97 million (11).

\section{CONCLUSIONS}

Despite the low incidence in pediatric aged, TB pericarditis remains a challenge due to both the non-specific symptoms in this age group and the difficulties related to the establishing the positive diagnosis and administering an adequate treatment. Nevertheless, the prognosis is favorable in the lack of complications. 


\section{REFERENCES}

1. Hatzenbuehler L.A., Starke J.R. Tuberculosis (Mycobacterium tuberculosis). In Kliegman R.M., Stanton B.F., St. Geme III J.W., Schor N.F. Nelson Texbook of Pediatrics, Edition 20. Philadelphia: Elsevier, 2016:1446-1462

2. Khan M.A., Mirza S.H., Abbasi S.A., Butt T., Anwar M. Peripheral blood-based polymerase chain reaction in diagnosis of pulmonary tuberculosis. J Ayub Med Coll Abbottabad 2006; 18(2):25-28.

3. Jenkins H.E., Yuen C.M., Rodriguez C.A. et al. Mortality in children diagnosed with tuberculosis: a systematic review and meta-analysis. Lancet Infect Dis 2016; pii: S1473-3099(16)30474-1. doi: 10.1016/S1473-3099(16)30474-1. [Epub ahead of print]

4. Jenkins H.E. Global Burden of Childhood Tuberculosis. Pneumonia (Nathan) 2016; 8:24.

5. Marais B.J., Gie R.P., Schaaf H.S. et al. The natural history of childhood intra-thoracic tuberculosis: a critical review of literature from the pre-chemotherapy era. Int J Tuberc Lung Dis 2004; 8(4):392-402.[PubMed]
6. Chiang S.S., Khan F.A., Milstein M.B. et al. Treatment outcomes of childhood tuberculous meningitis: a systematic review and meta-analysis. Lancet Infect Dis 2014; 14(10):947-57. [PubMed]

7. Didilescu C., Cioran N., Chiotan D., Popescu G. Tuberculosis in children in Romania. Pneumologia 2013; 62(1):10-4.

8. El Azbaoui S., Sabri A., Ouraini S. et al. Utility of the QuantiFERON@-TB Gold In-Tube assay for the diagnosis of tuberculosis in Moroccan children. Int J Tuberc Lung Dis 2016; 20(12):1639-1646.

9. Mazzola E., Arosio M., Nava A., Fanti D., Gesu G., Farina C. Performance of real-time PCR Xpert ${ }^{\circ} M T$ TB/RIF in diagnosing extrapulmonary tuberculosis. Infez Med 2016; 4(24):304-309.

10. Yuen C.M., Amanullah F., Dharmadhikari A. et al. Turning off the tap: stopping tuberculosis transmission through active case-finding and prompt effective treatment. Lancet 2015; 386(10010):2334-2343.

11. Houben R.M.G.J., Dodd P.J. The global burden of latent tuberculosis infection - a re-estimation. PLoS Med 2016; 13(10):e1002152. 